\title{
Dominios y facetas de la personalidad en estudiantes universitarios con patrón nocivo del consumo de alcohol
}

\author{
Ivonne L. Carpio T. \\ Unidad Coordinadora de Estudios no Presenciales, Universidad Católica de Cuenca, Av. de las \\ Américas y Humboldt, Cuenca, Ecuador, 010150. \\ Autor para correspondencia: ivoncarpiot@hotmail.com, icarpiot@ucacue.edu.ec \\ Fecha de recepción: 17 de marzo de 2015 - Fecha de aceptación: 10 de agosto de 2015
}

\section{RESUMEN}

El artículo presenta un análisis transversal de las facetas de personalidad de estudiantes universitarios con indicadores de consumo de riesgo y perjudicial de alcohol. El Alcohol Use Disorders Identificación Test (AUDIT) se utilizó para dividir un grupo no probabilístico aleatorio de 75 estudiantes, en un grupo de 40 estudiantes con patrones peligrosos y nocivos del consumo de alcohol y otro grupo de 35 estudiantes que no presenten estos síntomas. La personalidad de los individuos de ambos grupos se caracterizó mediante la prueba Inventario de Personalidad NEO Revisado (NEO PI$\mathrm{R})$, que mide seis facetas de cinco dimensiones de personalidad. Los resultados del grupo de estudiantes con alto consumo de alcohol mostraron una mayor prevalencia presente en los hombres (77.5\%), soleros (97.5\%), matriculados en el tercero y cuarto semestre (47.5\%), siendo la media de 21 años. Las diferencias significativas observadas entre los dos grupos fueron, en el dominio de personalidad de extraversión y en las facetas de hostilidad, gregarismo, búsqueda de emociones, emociones positivas, actividad, autodisciplina y deliberación.

Palabras clave: Estudiantes universitarios, abuso de alcohol, cinco grandes puntuaciones de la escala de personalidad, hostilidad, extraversión, responsabilidad.

\begin{abstract}
The manuscript presents a cross-sectional analysis of facets of the personality of college student's utilizing indicators of risk and damage related to alcohol consumption. The Alcohol Use Disorders Identification Test (AUDIT) was used to divide a randomly selected non-probabilistic group of 75 students in a group of 40 students with hazardous and harmful patterns of alcohol consumption and a group of 35 students not showing these symptoms. The personality of the individuals in both group were characterized using the Revised NEO Personality Inventory test (NEO PI-R), measuring six facets of the five personality dimension. Results of the group of students with high alcohol consumption show that a higher prevalence of alcohol misuse is present in men $(77.5 \%)$, single $(97.5 \%)$, enrolled in the third and fourth semester (47.5\%), being 21 years old on average. Significant differences were noticed between both groups in the personality domain of extraversion and facets of hostility, gregariousness, excitement-seeking, positive emotions, actions, self-discipline and deliberation.
\end{abstract}

Keywords: University students, alcohol abuse, big five scale scores of personality, hostility, extraversion, responsibility.

\section{INTRODUCCIÓN}

El alcohol, es una de las drogas más consumidas en el mundo y según datos de la Organización Mundial de la Salud, su consumo nocivo causa 2.5 millones de muertes cada año, siendo 
representativo en la población joven en edades comprendidas entre 15 y 29 años (320,000 jóvenes). El alcoholismo tiene una relación causal entre consumo y diferentes tipos de enfermedades y/o lesiones (Monteiro, 2007), además se asocia a comportamientos de alto riesgo, incluyendo sexo no seguro y uso de otras sustancias psicoactivas (OPS, 2007). Además, según Ramírez (2000) el uso excesivo influencia negativamente las relaciones sociales y familiares, como diversas formas de violencia familiar, conflictos maritales y abuso de menores. OPS (2000) refiere los efectos negativos del alcohol en absentismo laboral y la baja productividad económica. Borges et al. (2004) y Cherpitel \& Ye (2010) citan que el uso excesivo de alcohol es a menudo la causa de deserción escolar, admisiones en salas de emergencia, comportamiento violento, fatalidades en automovilistas y peatones cuando se conduce en estado de ebriedad.

El alcohol es considerado como el principal factor de riesgo para la carga de morbilidad; en América es de 5.4\% mientras que en el promedio mundial de 3.7\%, con índices más altos al promedio mundial (Monteiro, 2007). En América Latina y el Caribe existen alrededor de 30 millones de personas que pueden ser diagnosticadas con trastornos por el consumo de alcohol; sin embargo, más del 75\% de ellas no han recibido ninguna atención médica (OPS, 2007). Colombia tiene un consumo anual per cápita de 8.9 litros por bebedor, indicando un riesgo alto. En este país el consumo de alcohol es de un $86.1 \%$ al menos una vez en la vida, siendo mayor en los hombres que en las mujeres $(90 \%$ que equivale al 4.3 millones y $82 \%$ a 2.6 millones respectivamente) y el equivalente a 7 millones de personas con consumo en el último mes (Gobierno Nacional de la República de Colombia, 2008). De acuerdo con la misma fuente en la ciudad de Medellín y en el Área Metropolitana se reporta un 41\% de consumo en el último mes (993,527 de personas). Las edades comprendidas entre los 18 a 24 años, es el grupo poblacional que más ingiere dicha sustancia con un $46.3 \%$, seguido de personas con edades entre 25 a 34 años $\left(42.8 \%\right.$ ) y los estratos socioeconómicos ${ }^{1}$ de mayor incidencia son 5 (medio) y 6 (medio-alto) con el 56.9\%, cifra significativamente más alta que el estrato 4 (medio) que tiene un porcentaje de $38.1 \%$.

Lo que indica que "poseemos una problemática acuciante con serias implicaciones para el bienestar de la población, la salud pública, la convivencia ciudadana, la seguridad y el futuro de las nuevas generaciones" (Gobierno Nacional de la República de Colombia, 2008: 146); por lo tanto es importante realizar investigaciones que ayuden a comprender lo relacionado con el fenómeno del consumo de alcohol, para poder intervenirlo de una manera más eficaz en pro de la salud mental. Existen diferentes tipos de ingesta, según los gramos de alcohol consumido, en forma de unidades de bebida estándar a la que se le suma la cantidad de la ingesta, el tiempo y la frecuencia del consumo, entre los que se encuentra, el consumo de riesgo que es regular con alrededor de 20 a $40 \mathrm{~g}$ diarios de alcohol en mujeres y de 40 a $60 \mathrm{~g}$ en varones, siendo un patrón de ingesta que aumenta el riesgo de consecuencias adversas para el bebedor y otras personas (Anderson et al., 2008).

El perjudicial definido por el CIE-10 como, un patrón de consumo de sustancias psicoactivas que está causando daños a la salud, física o mental (OMS, 2001), una ingesta promedio de más de $40 \mathrm{~g}$ de alcohol al día en mujeres y de más de $60 \mathrm{~g}$ en hombres, que conlleva a consecuencias tanto para la salud física, mental y social, para sí mismo como para los demás. La dependencia al alcohol, cursa con un deseo intenso y dificultad para controlar el consumo, con persistencia a pesar de las consecuencias perjudiciales, aumento de la tolerancia al alcohol y abstinencia física cuando se interrumpe el consumo que se asocia a un conjunto de fenómenos conductuales, cognitivos y fisiológicos que aparecen deteriorando la salud mental, física, las relaciones interpersonales y el funcionamiento social o económico (Babor et al., 2001; Anderson et al., 2008). La identificación de los tipos de consumo es clave para la intervención por lo que la OMS (2001), propone el test AUDIT (Alcohol Use Disorders Identificación Test) que permite educar a los pacientes sobre la cantidad y frecuencia de la ingesta, establece el diagnóstico del estado actual del paciente, así como también puede alertar a los clínicos sobre la necesidad de advertir a las personas cuyo consumo podría afectar adversamente a su medicación y a otros aspectos de su tratamiento (Babor et al., 2001).

\footnotetext{
${ }^{1}$ La estratificación socioeconómica en Colombia: https://es.wikipedia.org/wiki/Estratificaci\%C3\%B3n _socioecon\%C3\%B3mica_en_Colombia\#Estratos_socioecon.C3.B3micos_en_Colombia.
} 
La personalidad es una de las variables psicológicas más relevantes en el uso y abuso de alcohol (Cloninger, 1987). Esta investigación utiliza el modelo de los cinco grandes ("big five"), ya que identifica las características que distinguen a las personas entre sí, estableciendo una organización de la personalidad compuesta de seis rasgos particulares en cada una de las dimensiones de neuroticismo, extraversión, apertura a la experiencia, amabilidad y responsabilidad (Belloch et al., 2009), que se evalúan a través de la prueba Revised NEO Personality Inventory Test (NEO PI-R) creado por Costa y McCrae (1992). El modelo de los cinco grandes es utilizado en múltiples estudios y se encuentran en perfecta consonancia con otros métodos de investigación, así como a través del tiempo, los contextos y culturas (McAdams \& Donnellan, 2009).

Los factores asociados al consumo de alcohol pueden ser de carácter biológico, psicológico y social (Ortiz de Zarate et al., 2011). Dentro de los componentes psicológicos de riesgo en estudiantes, se presenta el neuroticismo, niveles bajos de afrontamiento a la ansiedad y depresión, además de puntuaciones bajas en responsabilidad (Raynor \& Levine, 2009). Cyders et al. (2009) menciona que la búsqueda de sensaciones, falta de planeación y perseverancia aumentan la frecuencia de consumo. En las dimensiones de personalidad que pueden predecir el consumo está la extroversión, baja responsabilidad y amabilidad (Hong \& Paunonen, 2009; Mezquita et al., 2009), con niveles altos de antagonismo, impulsividad, alexitimia y emociones negativas (Gunnarsson et al., 2008; Mezquita et al., 2009).

El ámbito universitario se convierte en una búsqueda intelectual y crecimiento personal, que moldea la identidad de cada persona y en el que se enfrenta a situaciones estresantes, por lo que el consumo de sustancias psicoactivas se realiza como un "facilitador del vínculo social, el encuentro con el otro, la necesidad de identificarse y diferenciarse a la vez, propiciando una desinhibición que lleva al relajamiento, diversión y creación de lazos sociales" (Red Unir, 2009: 158; Wartski et al., 2011). Los motivos principales para el consumo de alguna sustancia psicoactiva son curiosidad 83.9\%, facilidad de acceso 38.6\% y presión de amigos 11.2\% (Tirado et al., 2009). En el 2001 la encuesta sobre el consumo de sustancias psicoactivas, encontró que en todas las ciudades de Colombia, existe un mayor consumo de alcohol en estudiantes universitarios (RUMBOS, 2002). Ellos presentan riesgo de consumo moderado, tanto en situaciones personales (88.2\%), como en aquellas que involucran a otras personas (98.4\%) y los mayores índices de riesgo se evidencian cuando se asocian emociones agradables, emociones desagradables, conflictos con los otros, tener un amigo consumidor y la presión social para beber (Albarracín Ordoñez \& Muñoz Ortega, 2008; Cruz Díaz et al., 2011; Tirado et al., 2009).

Es así como en la investigación se buscó conocer si existe diferencias entre los dominios y facetas de personalidad en estudiantes universitarios con un consumo de riesgo y/o perjudicial de alcohol y estudiantes sin dicha ingesta. Esto con el fin de ofrecer datos que favorezcan programas futuros en la formación integral de los estudiantes y la comunidad universitaria, mediante acciones de educación (prevención y promoción) aplicables que permitan minimizar el daño que causa esta problemática (Wartski et al., 2011).

\section{MATERIALES Y MÉTODOS}

\section{Participantes}

Se seleccionaron estudiantes de la carrera de ingeniería de una universidad privada de la ciudad de Medellín en Colombia, mayores de edad, con consentimiento informado y voluntariedad para participar.

\section{Tamaño y precisión de la muestra}

Se realizó un muestreo no probabilístico aleatorio, en el que participaron 75 personas, 24 mujeres y 51 varones, la división de los grupos de estudio se llevó a cabo a través de la prueba AUDIT, en la cual un puntaje mayor a 8 representaba un consumo de riesgo y mayor a 16 un consumo perjudicial de 
alcohol. El primero grupo estuvo conformado por 40 estudiantes universitarios con un consumo de riesgo y/o perjudicial y el segundo por 35 estudiantes que no presentan dicho consumo.

\section{Mediciones}

Se utilizó un cuestionario para medir las variables sociodemográficas; el test AUDIT que permite un tamizaje en relación al nivel de consumo, desde el rango de sin riesgo hasta la ingesta problemática y perjudicial de alcohol; el cual es un instrumento de uso internacional, medido en una escala tipo Likert de 0 a 4, que se categoriza de acuerdo a la cantidad y frecuencia de la ingesta de alcohol; con sensibilidad del $80 \%$ y especificidad del 90\% (Babor et al., 2001; Monteiro, 2007).

El test NEO PI-R evalúa cinco dimensiones de la personalidad, las cuales son: neuroticismo, extraversión, amabilidad, apertura y responsabilidad, y seis facetas o subdimensiones en cada factor. En el dominio de neuroticismo se encuentra: ansiedad, hostilidad, depresión, ansiedad social, impulsividad y vulnerabilidad; en extraversión: cordialidad, gregarismo, asertividad, actividad, búsqueda de emociones, y emociones positivas; en apertura a las nuevas experiencias: fantasía, estética, sentimientos, acciones, ideas y valores; en el dominio de amabilidad: confianza, franqueza, altruismo, actitud conciliadora, modestia y sensibilidad con los demás; y por último en responsabilidad: competencia, orden, sentido del deber, necesidad de logro, autodisciplina y deliberación (Brody \& Ehrlichman, 2000; Trull \& Phares, 2003). El test está formado de 240 reactivos tipo Likert de 0 a 4 que van desde desacuerdo, a totalmente de acuerdo, con buenos niveles de consistencia interna para las escalas de dominios y facetas, con una confiabilidad re-test (Costa \& McCrae, 1992).

\section{Diseño de investigación}

Es un estudio cuantitativo, descriptivo, comparativo de corte transversal.

\section{Contexto donde se hizo la investigación}

La investigación se llevó a cabo con estudiantes que cursan la carrera de ingeniería en una universidad privada de la ciudad de Medellín, de acuerdo a los parámetros exigidos para tal fin como la aprobación escrita institucional y el aval ético del comité respectivo.

\section{Análisis de la información}

El análisis de la información se realizó mediante los programas Microsoft Office Excel 2010 y el programa Statgraphics Centurion XVI; la información se consignó en tablas de acuerdo a los resultados arrojados en estos programas. Para las variables sociodemográficas cualitativas y de consumo de alcohol se realizó el análisis descriptivo, mediante una distribución de frecuencias y para las cuantitativas como edad, edad de inicio y tiempo de consumo se consideraron medidas de tendencia central y de variabilidad. El análisis de los dominios y facetas de personalidad se realizó mediante una prueba de diferencia de proporciones que permite encontrar diferencias significativas entre sí, con un nivel de significancia del 5\%. Se categorizaron los puntajes en tres niveles, alto, promedio y bajo, recordando que el nivel promedio en los sujetos denota un nivel normal de dicha faceta o dominio, y los puntajes que se discutirán son los valores significativamente altos o bajos.

\section{RESULTADOS}

Los resultados obtenidos en la investigación se presentan en relación al tipo de consumo, variables sociodemográficas y personalidad. Se observó que el $45.3 \%$ presentó un consumo de riesgo y el $8 \%$ un consumo perjudicial con una posible dependencia al alcohol. Del grupo de consumo de riesgo y/o perjudicial (40 estudiantes; $53.3 \%$ de la población), el 15\% obtuvo un consumo perjudicial de alcohol con una posible dependencia y el $85 \%$ restante consumo de riesgo (Tabla 1). 
Las variables sociodemográficas en el grupo total muestran, que el $68 \%$ eran de sexo masculino y el $32 \%$ femenino, de estado civil soltero (96\%), estrato socioeconómico 5 (35\%), que vive con los padres $(80 \%)$ y que cursan el tercer y cuarto semestre académico (41\%).

En el grupo A (estudiantes con consumo de riesgo y/o perjudicial de alcohol) mostró que el $77.5 \%$ fueron hombres y el $22.5 \%$ mujeres. Los datos más relevantes en este grupo fueron el estado civil soltero (97.5\%), el nivel que cursan es tercer y cuarto semestre académico (47.5\%), el estrato socioeconómico 4 (35\%), y con quien viven los estudiantes de este grupo fue, con los padres $(72.5 \%)$, con un familiar (15\%), y solos (12.5\%); mientras que el grupo B (sin consumo) el $57.1 \%$ fueron hombres y $42.9 \%$ mujeres, cursan el quinto y sexto semestre (37.1\%), con un estrato socioeconómico cinco $(40 \%)$, y el $88.6 \%$ vive con los padres y el $11.4 \%$ con un familiar (Tabla 2 ).

Tabla 1. Tipo de consumo.

\begin{tabular}{ll}
\hline Clase de consumo & $\mathrm{N}(\%)$ \\
\hline Sin consumo & $35(46.7)$ \\
Consumo de riesgo & $34(45.3)$ \\
Consumo perjudicial / posible dependencia & $6(8)$ \\
\hline Total & $75(100)$ \\
\hline
\end{tabular}

Tabla 2. Características sociodemográficas.

\begin{tabular}{llll}
\hline \multirow{2}{*}{ Variables } & & \multicolumn{2}{c}{ Consumo de riesgo y/o perjudicial } \\
\cline { 3 - 4 } & & $\mathrm{Con}^{\mathrm{a}} \mathrm{N}(\%)$ & $\operatorname{Sin}^{\mathrm{b}} \mathrm{N}(\%)$ \\
\hline Sexo & Femenino & $9(22.5)$ & $15(42.9)$ \\
& Masculino & $31(77.5)$ & $20(57.2)$ \\
\hline Estado civil & Soltero & $39(97.5)$ & $33(94.3)$ \\
& Casado / unión libre & $1(2.5)$ & $2(5.7)$ \\
\hline Estrato & 2 & $-(-)$ & $1(2.9)$ \\
socioeconómico & 3 & $10(25)$ & $10(28.6)$ \\
& 4 & $14(35)$ & $6(17.1)$ \\
& 5 & $12(30)$ & $14(40)$ \\
& 6 & $4(10)$ & $4(11.4)$ \\
\hline Personas con & Con los padres & $29(72.5)$ & $31(88.6)$ \\
quien vive & Con un familiar & $6(15)$ & $4(11.4)$ \\
& Solo & $5(12.5)$ & $-(-)$ \\
\hline Semestre & 1 y 2 & $7(17.5)$ & $5(14.3)$ \\
académico & 3 y 4 & $19(47.5)$ & $12(34.3)$ \\
& 5 y 6 & $4(10)$ & $13(37.1)$ \\
& 7 y 8 & $3(7.5)$ & $3(8.6)$ \\
& 9 y 10 & $7(17.5)$ & $2(5.7)$ \\
\hline
\end{tabular}

Leyenda: ${ }^{a} \mathrm{~N}=40,{ }^{\mathrm{b}} \mathrm{N}=35$

En relación a la edad se evidenció que la media del grupo A fue de 21 años, mostrando una diferencia significativa y según el coeficiente de variación una homogeneidad de los datos. La edad promedio de inicio de ingesta de alcohol en los grupos fue de alrededor 13 años, siendo menor en el grupo A (11 años), mientras que en el otro grupo hubo quien no había consumido hasta el momento del estudio. El tiempo de ingesta de alcohol en el grupo A fue de 7 años, mientras que en el grupo B fue de 5 años, mostrando una diferencia significativa y una heterogeneidad de los datos (Tabla 3 ). 
Tabla 3. Comparación de medias en relación con la edad.

\begin{tabular}{|c|c|c|c|c|c|c|}
\hline \multirow{2}{*}{ Variables } & \multicolumn{5}{|c|}{ Con $^{\mathrm{a}}$} & \\
\hline & Media & DE & Min & $\operatorname{Max}$ & $\mathrm{CV}$ & \\
\hline Edad & 21.1 & 2.57 & 18 & 27 & $12.2 \%$ & \\
\hline Edad de inicio & 13.9 & 1.31 & 11 & 16 & $9.5 \%$ & \\
\hline Tiempo de consumo & 7.2 & 2.72 & 3 & 14 & $37.9 \%$ & \\
\hline \multirow{2}{*}{ Variables } & \multicolumn{5}{|c|}{$\operatorname{Sin}^{\mathrm{b}}$} & \multirow{2}{*}{$\mathrm{p}$} \\
\hline & Media & $\mathrm{DE}$ & Min & Max & $\mathrm{CV}$ & \\
\hline Edad & 19.5 & 1.44 & 18 & 23 & $7.4 \%$ & $0.001 *$ \\
\hline Edad de inicio & 13.8 & 2.94 & 0 & 18 & $21.4 \%$ & 0.803 \\
\hline Tiempo de consumo & 5.3 & 2.35 & 0 & 11 & $44.8 \%$ & $0.001 *$ \\
\hline
\end{tabular}

Leyenda: ${ }^{a} \mathrm{~N}=40,{ }^{b} \mathrm{~N}=35,{ }^{*} \mathrm{p}<0.05$

Los valores que se consideraron en la variable de personalidad son los que puntuaron en el nivel alto o bajo, ya que indica extremos, es decir fuera de lo normal. En la muestra total $(\mathrm{N}=75)$ las dimensiones de personalidad que fueron representativas son el neuroticismo con puntajes altos y niveles bajos en extroversión, amabilidad, apertura y responsabilidad. El grupo A presentó un mayor puntaje en la faceta de hostilidad; además en el dominio de extroversión, y las facetas de gregarismo, emociones positivas, búsqueda de emociones y actividad mostraron puntajes menores. En el dominio de responsabilidad, las facetas de deliberación y autodisciplina se enmarca también la diferencia; siendo bajo en los estudiantes con un consumo de riesgo o perjudicial. El grupo B presentó menores valores en la faceta de ansiedad; en los dominios de apertura y amabilidad no se encontraron diferencias significativas (Tabla 4).

\section{DISCUSIÓN}

De acuerdo a los resultados obtenidos en las variables sociodemográficas para el grupo de consumo de riesgo y/o perjudicial, la muestra describe un mayor número de estudiantes de sexo masculino, solteros, viven principalmente con sus padres, aunque hay un porcentaje que vive solo por lo que pudiera considerarse a la familia como factor protector. Además cursan tercero y cuarto semestre, de estrato socioeconómico 4, con una edad promedio de 21 , un inicio de consumo a la edad de 13 y una media de tiempo de ingesta de 7 años. Los datos reportados concuerdan con el estudio realizado en la ciudad de Medellín y en el área Metropolitana, donde existe un mayor consumo de alcohol en estudiantes universitarios (RUMBOS, 2002); con un incremento de ingesta en hombres con edades entre los 18 a 24 años (Cyders et al., 2009); y se diferencia en relación al estrato socioeconómico cuya mayor incidencia fue el 5 y 6 (Gobierno Nacional de la República de Colombia, 2008).

Con respecto a la personalidad, el dominio de neuroticismo mostró puntajes altos en ambos grupos, caracterizado por afectos negativos como miedo, venganza, culpa, sin capacidad de autocontrol y afrontamiento al estrés (Mezquita et al., 2009). El presente estudio evidenció diferencias significativas en las facetas de hostilidad y ansiedad; los estudiantes que presentan un consumo de riesgo y/o perjudicial son más agresivos, con tendencia a experimentar ira, frustración y rencor (Mezquita et al., 2010).

El dominio extroversión es menor en el grupo A, es decir son personas reservadas, independientes, tienen preferencia por la soledad, donde las facetas de gregarismo, búsqueda de emociones, emociones positivas y actividad se muestran igualmente bajas, lo que implica rechazo a los estímulos sociales, poca intensidad emocional y presencia de estados emocionales negativos como 
MASKANA, Vol. 6, No. 2, 2015

Tabla 4. Comparación de los dominios y facetas de personalidad en estudiantes con y sin consumo de riesgo y perjudicial de alcohol.

\begin{tabular}{|c|c|c|c|c|c|c|c|c|c|}
\hline \multirow{3}{*}{ Facetas de personalidad } & \multicolumn{6}{|c|}{ Consumo de riesgo y/o perjudicial de alcohol } & \multirow{2}{*}{\multicolumn{3}{|c|}{$\mathrm{p}$}} \\
\hline & \multicolumn{3}{|c|}{ Con $^{\mathrm{a}}$} & \multicolumn{3}{|c|}{$\operatorname{Sin}^{\mathrm{b}}$} & & & \\
\hline & Alta & Promedio & Bajo & Alta & Promedio & Bajo & Alta & Promedio & Bajo \\
\hline Neuroticismo & 0.85 & 0.13 & 0.03 & 0.89 & 0.06 & 0.06 & 0.553 & $0.000 *$ & 0.222 \\
\hline Ansiedad & 0.33 & 0.53 & 0.15 & 0.29 & 0.43 & 0.29 & 0.714 & 0.262 & $0.030 *$ \\
\hline Hostilidad & 0.90 & 0.08 & 0.03 & 0.77 & 0.23 & 0.00 & $0.013^{*}$ & $0.000^{*}$ & 0.311 \\
\hline Depresión & 0.73 & 0.25 & 0.03 & 0.86 & 0.09 & 0.06 & 0.074 & $0.020 *$ & 0.222 \\
\hline Vulnerabilidad & 1.00 & 0.00 & 0.00 & 0.91 & 0.09 & 0.00 & 0.070 & 0.070 & 1.000 \\
\hline Impulsividad & 0.36 & 0.40 & 0.28 & 0.49 & 0.34 & 0.17 & 0.113 & 0.496 & 0.165 \\
\hline Ansiedad social & 0.60 & 0.35 & 0.05 & 0.60 & 0.34 & 0.06 & 1.000 & 0.931 & 0.843 \\
\hline Extroversión & 0.00 & 0.00 & 1.00 & 0.06 & 0.11 & 0.83 & 0.145 & $0.033 *$ & $0.007 *$ \\
\hline Cordialidad & 0.00 & 0.00 & 1.00 & 0.00 & 0.03 & 0.97 & 1.000 & 0.312 & 0.306 \\
\hline Gregarismo & 0.13 & 0.35 & 0.55 & 0.23 & 0.43 & 0.34 & 0.071 & 0.340 & $0.015 *$ \\
\hline Asertividad & 0.05 & 0.53 & 0.43 & 0.11 & 0.43 & 0.46 & 0.085 & 0.262 & 0.707 \\
\hline Emociones positivas & 0.00 & 0.05 & 0.95 & 0.00 & 0.14 & 0.86 & 1.000 & $0.014 *$ & $0.014 *$ \\
\hline Búsqueda de emociones & 0.03 & 0.15 & 0.83 & 0.09 & 0.40 & 0.51 & $0.026^{*}$ & $0.000 *$ & $0.000 *$ \\
\hline Actividad & 0.03 & 0.30 & 0.68 & 0.03 & 0.54 & 0.43 & 0.887 & $0.002 *$ & $0.002 *$ \\
\hline Amabilidad & 0.00 & 0.00 & 1.00 & 0.03 & 0.06 & 0.91 & 0.236 & 0.645 & 0.070 \\
\hline Actitud conciliadora & 0.33 & 0.35 & 0.33 & 0.29 & 0.37 & 0.34 & 0.057 & 0.794 & 0.825 \\
\hline Modestia & 0.05 & 0.35 & 0.60 & 0.09 & 0.29 & 0.63 & 0.553 & 0.428 & 0.734 \\
\hline Sensibilidad a los demás & 0.05 & 0.03 & 0.93 & 0.00 & 0.14 & 0.83 & 0.147 & $0.000 *$ & 0.132 \\
\hline Confianza & 0.00 & 0.23 & 0.78 & 0.03 & 0.20 & 0.77 & 0.748 & 0.723 & 0.960 \\
\hline Franqueza & 0.08 & 0.25 & 0.68 & 0.03 & 0.20 & 0.77 & 0.081 & 0.493 & 0.226 \\
\hline Altruismo & 0.00 & 0.00 & 1.00 & 0.00 & 0.03 & 0.97 & 1.000 & 0.312 & 0.300 \\
\hline
\end{tabular}

Leyenda: Para un mejor análisis los resultados del test NEO PI-R aplicados a los estudiantes, se agruparon en tres niveles según las puntuaciones obtenidas en cada dominio o facetas, alto, promedio (normal) y bajo. Las siglas, p alto, p promedio y p bajo, que aparecen al final de la tabla, hacen referencia al resultado de la prueba de comparación de proporciones en relación a los dominios y facetas de personalidad de estudiantes con y sin un consumo de riesgo y/o perjudicial de alcohol, con un nivel de significancia del $5 \%$. ${ }^{\mathrm{a}} \mathrm{N}=40,{ }^{\mathrm{b}} \mathrm{N}=35,{ }^{*} \mathrm{p}<0.05$. 
MASKANA, Vol. 6, No. 2, 2015

Tabla 4. Comparación de los dominios y facetas de personalidad en estudiantes con y sin consumo de riesgo y perjudicial de alcohol (continuación).

\begin{tabular}{|c|c|c|c|c|c|c|c|c|c|}
\hline \multirow{3}{*}{ Facetas de personalidad } & \multicolumn{6}{|c|}{ Consumo de riesgo y/o perjudicial de alcohol } & \multirow{2}{*}{\multicolumn{3}{|c|}{$\mathrm{p}$}} \\
\hline & \multicolumn{3}{|c|}{ Con $^{\mathrm{a}}$} & \multicolumn{3}{|c|}{$\operatorname{Sin}^{\mathrm{b}}$} & & & \\
\hline & Alta & Promedio & Bajo & Alta & Promedio & Bajo & Alta & Promedio & Bajo \\
\hline Apertura & 0.03 & 0.08 & 0.90 & 0.00 & 0.03 & 0.97 & 0.312 & 0.275 & 0.138 \\
\hline Fantasía & 0.05 & 0.20 & 0.75 & 0.03 & 0.26 & 0.71 & 0.545 & 0.405 & 0.141 \\
\hline Estética & 0.15 & 0.48 & 0.38 & 0.17 & 0.43 & 0.40 & 0.724 & 0.589 & 0.825 \\
\hline Sentimientos & 0.03 & 0.05 & 0.93 & 0.03 & 0.06 & 0.91 & 0.977 & 1.000 & 0.861 \\
\hline Valores & 0.00 & 0.03 & 0.98 & 0.00 & 0.00 & 1.00 & 1.000 & 0.936 & 0.297 \\
\hline Ideas & 0.05 & 0.10 & 0.85 & 0.00 & 0.20 & 0.80 & 0.147 & 0.054 & 0.412 \\
\hline Acciones & 0.15 & 0.43 & 0.43 & 0.14 & 0.37 & 0.49 & 0.906 & 0.528 & 0.474 \\
\hline Responsabilidad & 0.00 & 0.00 & 1.00 & 0.00 & 0.00 & 1.00 & 1.000 & 1.000 & 1.000 \\
\hline Competencia & 0.00 & 0.03 & 0.98 & 0.00 & 0.00 & 1.00 & 1.000 & 0.936 & 0.311 \\
\hline Orden & 0.03 & 0.33 & 0.65 & 0.00 & 0.23 & 0.77 & 0.312 & 0.226 & 0.132 \\
\hline Sentido del deber & 0.00 & 0.00 & 1.00 & 0.00 & 0.00 & 1.00 & 1.000 & 1.000 & 1.000 \\
\hline Deliberación & 0.15 & 0.40 & 0.45 & 0.06 & 0.23 & 0.71 & 0.109 & $0.038 *$ & $0.001 *$ \\
\hline Autodisciplina & 0.00 & 0.20 & 0.80 & 0.00 & 0.00 & 1.00 & 1.000 & $0.001 *$ & $0.001 *$ \\
\hline Necesidad de logro & 0.00 & 0.08 & 0.92 & 0.00 & 0.03 & 0.97 & 1.000 & 0.275 & 0.123 \\
\hline
\end{tabular}

Leyenda: Para un mejor análisis los resultados del test NEO PI-R aplicados a los estudiantes, se agruparon en tres niveles según las puntuaciones obtenidas en cada dominio o facetas, alto, promedio (normal) y bajo. Las siglas, $\mathrm{p}$ alto, $\mathrm{p}$ promedio y p bajo, que aparecen al final de la tabla, hacen referencia al resultado de la prueba de comparación de proporciones en relación a los dominios y facetas de personalidad de estudiantes con y sin un consumo de riesgo y/o perjudicial de alcohol, con un nivel de significancia del $5 \%$. ${ }^{\mathrm{a}} \mathrm{N}=40,{ }^{\mathrm{b}} \mathrm{N}=35,{ }^{*} \mathrm{p}<0.05$ 
tristeza (Costa \& McCrae, 1992). Esas observaciones se corresponden muy bien con los resultados de McAdams \& Donnellan (2009) en estudiantes universitarios de primer año. En investigaciones con colegiales refieren como predictor de aumento de ingesta, la extroversión (Raynor \& Levine, 2009; Mezquita et al., 2009), aunque Mezquita et al. (2010) refieren que es necesario profundizar más en este aspecto debido a que el consumo de alcohol es un medio para la desinhibición y favorece al contacto social, las personas que tienen bajo afrontamiento a la ansiedad y dificultades en relaciones sociales presentaron un consumo alto de alcohol como motivante interno, lo que permite reflexionar sobre los datos obtenidos.

Por su parte las facetas de vulnerabilidad y ansiedad social puntuaron alto en ambos grupos. Esto describió la muestra $(\mathrm{N}=75)$ como personas que se sienten incómodos con los demás, sensibles a hacer el ridículo, propensos a sentimientos de inferioridad, incapaces de luchar contra el estrés, dependientes y desesperanzados en situaciones críticas.

El dominio de apertura implica la búsqueda activa de nuevas experiencias, curiosidad e imaginación y el de amabilidad permite una calidad en las interacciones personales basados en la confianza, ayuda y altruismo, éstas no muestran diferencias significativas; sin embargo, con puntajes bajos, es decir, los participantes de este estudio son centrados en sí mismos, reticentes a involucrarse en los problemas de los demás, con embotamiento emocional por la no importancia que le dan a los afectos. La faceta de amabilidad se asoció en otras investigaciones a un aumento de consumo, y conductas de riesgo (Mezquita et al., 2009; Hong \& Paunonen, 2009).

El dominio de responsabilidad mostró puntajes bajos en todos los estudiantes, lo que denota poco grado de organización, persistencia y control en los comportamientos orientados hacia metas específicas. Las facetas que hacen parte de este dominio y que puntuaron con diferencias significativas en los estudiantes con consumo de riesgo y/o perjudicial fueron autodisciplina y deliberación las cuales fueron bajas, lo que implica que son personas que postergan el inicio de sus labores y responsabilidades, poco perseverantes, precipitados para hablar y actuar, sin tener en cuenta las consecuencias, aunque son capaces de tomar decisiones inmediatas cuando es necesario. Estos resultados concuerdan con otras investigaciones que refieren que a menor responsabilidad aumenta el consumo (Cyder et al., 2009; Mezquita et al., 2009; Raynor \& Levine, 2009; Hong \& Paunonen, 2009; Mezquita et al., 2010).

\section{CONCLUSIONES}

El propósito de este estudio fue describir los dominios y facetas de personalidad en estudiantes universitarios con diferentes niveles de consumo de alcohol. Los resultados que se obtuvieron en estudiantes con un consumo de riesgo y/o perjudicial de alcohol fue, una mayor prevalencia en hombres, de estado civil solteros, con una edad promedio de 21 años. En su mayoría cursan tercer y cuarto semestre académico, y se encontró diferencias significativas en el dominio de extroversión y en las facetas de hostilidad, ansiedad, gregarismo, búsqueda de emociones, emociones positivas, actividad, autodisciplina y deliberación.

Las limitaciones que presentó el estudio fue que se realizó únicamente con población universitaria de la carrera de ingeniería; sin embargo nos permitió entender y conocer las dimensiones y facetas de personalidad en jóvenes universitarios con indicadores de consumo de riesgo y/o perjudicial de alcohol que según investigaciones previas, es la población que presenta mayor consumo de alcohol (Cruz Díaz et al., 2011). Sería importante investigar en una muestra más grande para poder conocer las diferentes variables psicológicas que influyen, así como los factores de riesgo y protectores en el consumo, además de la utilización de estos datos para la creación de proyectos de prevención cuya pretensión será impactar en la ingesta de alcohol que es una problemática de salud pública mundial. 


\section{REFERENCIAS}

Albarracín Ordoñez, M., L. Muñoz Ortega, 2008. Factores asociados al consumo de alcohol en estudiantes de los dos primeros años de carrera universitaria. Revista Liberabit, Lima (Perú), 14, 49-61.

Anderson, P., A. Gual, J. Colon, 2008. Alcohol y atención primaria de la salud: Informaciones clínicas básicas para la identificación y el manejo. Organización Panamericana de la Salud, 148 pp. Recuperado de http://www.who.int/substance_abuse/publications/alcohol_atencion _primaria.pdf el 23 de abril de 2012.

Babor, F.T., J.C. Higgins-Biddle, J.B. Saunders, M.G. Monteiro, 2001. Cuestionario de identificación de los trastornos debidos al consumo de alcohol. WHO/MSD/MSB/01.6a, 60 pp. Disponible en http://www.who.int/substance_abuse/activities/en/AUDITmanualSpanish.pdf/.

Belloch, A., B. Sandín, F. Ramos, 2009. Manual de psicopatología. Volumen 1 (Edición revisada). The McGraw-Hill Companies, 462 pp. Disponible en http://www.academia.edu/ 7474998/Manual_de_psicopatolog\%C3\%ADa._Amparo_Belloch_Bonifacio_Sand\%C3\%ADn_F rancisco_Ramos.

Borges, G., C.J. Cherpitel, M.E. Medina-Mora, L. Mondragon, 2004. Violence related injuries in the emergency room: alcohol, depression, and conduct problems. Subst. Use Misuse, 39(6), 911-930.

Brody, N., H. Ehrlichman, 2000. Psicologia de la personalidad. Madrid: Prentice Hall.

Cherpitel, C.J., Y. Ye, 2010. Alcohol and violence-related injuries among emergency room patiens in an international perspective. J. Am. Psychiatr. Nurses Assoc., 16, 227-235.

Cloninger, C.R., 1987. Neurogenetic adaptive mechanisms in alcoholism. Science, 236, 410-416.

Costa, P.T., R.R. McCrae, 1992. Four ways five factors are basic. Person. individ. Diff., 13(6), 653665.

Cruz Díaz, A.B., Y. Luna Ramírez, M.Y. Méndez Bernal, T. Muñoz Torres, A. Nava Medina, M. Adán Plata, A. Rodríguez Martínez, G. Reséndiz Gutiérez, R. Rocha Rodríguez, M.I. Maldonado Cervantes, 2011.Factores asociados al consumo de alcohol en estudiantes de ingeniería civil. Psicología y Salud, 21(2), 265-271.

Cyders, M.A., K. Flory, S. Rainer, G.T. Smith, 2009. The role of personality dispositions to risky behavior in predicting first year college drinking. Addiction, 104(2), 193-202.

Gobierno Nacional de la República de Colombia, 2008. Estudio Nacional de Sustancias Psicoactivas en Colombia. Recuperado de http://www.odc.gov.co/Portals/1/comision_asesora/docs/ CO03272008-estudio-nacional-consumo-sustancias-psicoactivas-colombia-2008-.pdf el 15 de marzo de 2012.

Gunnarsson, M., J.P. Gustavsson, A. Tengström, J. Franck, C. Fahlke, 2008. Personality traits and their associations with substance use among adolescents. Person. individ. Diff., 45(5), 356-360.

Hong, R.Y., S.V. Paunonen, 2009. Personality traits and health-risk behaviours in university students. Eur. J. Personality, 23(8), 675-696.

McAdams, K., B. Donnellan, 2009.Facets of personality and drinking in first-year college students. Personality and Individual Differences, 46(2), 207-212.

Mezquita, L., E. Maestre, H. Mestre, M. Viñas, J. Moya, G. Ortet, 2009. Relación entre personalidad y consumo de alcohol en adolescentes españoles y escoceses. Universität Jaume I, Jornades de Foment de la Investigació, 12 pp. Disponible en http://www.uji.es/bin/publ/edicions/jfi11/2.pdf.

Mezquita, L., Stewart, S., Á. Ruipérez, 2010. Big-five personality domains predict internal drinking motives in young adults. Personality and Individual Differences, 49(3), 240-245.

Monteiro, M.G., 2007. Alcohol y salud publica en las Americas. Un caso para la acción. OPS/OMS, Washington DC, EE.UU., 64 pp. Disponible en http://www.who.int/substance_abuse/ publications/alcohol_public_health_americas_spanish.pdf. 
OMS, 2001. Carga de los trastornos mentales y conductuales. Informe sobre la salud en el mundo: Capítulo 2, 26 pp. Disponible en http://www.who.int/whr/2001/en/whr01_ch2_es.pdf.

OPS, 2000. Guía internacional para vigilar el consumo de alcohol y sus consecuencias sanitarias. Organización Panamericana de la Salud, 193 pp. Disponible en http://www.who.int/substance_ abuse/publications/monitoring_alcohol_consumption_spanish.pdf.

OPS, 2007. Salud en las Américas. Organización Panamericana de la Salud, Publicación Científica y Técnica No. 622, Volumen 1- Regional (482 pp.) y Volumen 2 - Países (812 pp). Disponible en http://www.bvs.hn/ docum/ops/SA2007/SAvol1esp/SEA07\%20Regional\%20SPA.pdf.

Ortiz de Zarate, A., I. Alonso, A. Ubis, M.A. Ruiz de Azúa, 2011. Guías de cuidados de enfermería en salud mental. Vol. 6: Adicciones ( $1^{\mathrm{a}}$ ed.), 223 pp. Edición: Elsevier Masson, Barcelona, España.

Ramírez, C., 2000. Co-ocurrencia de comportamientos violentos y adictivos en jóvenes y adultos en ciudades Colombianas. Acta Colombiana de Psicología, 63-78.

Raynor, D.A., H. Levine, 2009. Associations between the five-factor model of personality and health behaviors among college students. J. Am. Coll. Health, 58(1), 73-81.

Red Unir, 2009. Del viaje en U. Red para la Investigación, la Formación y la Prevención del Consumo de Sustancias Psicoactivas en el Ámbito Universitario. Medellin, Colombia.

RUMBOS, 2002. Juventud y consumo de sustancias psicoactivas: resultados de la encuesta nacional de 2001 en jóvenes escolarizados de 10 a 24 años. Bogotá, Colombia: Presidencia de la República, 296 pp. Disponible en http://www.mamacoca.org/docs_de_base/Consumo/ Encuesta_consumo_SPA_2001.pdf.

Tirado, A.F., M. Álvarez, J.D. Velásquez, L. Gómez, C. Ramírez, A.R. Vargas, 2009. Prevalencia y factores de riesgo para el consumo y dependencia de drogas en estudiantes de una universidad de Medellín, Colombia, 2009. Rev. Fac. Nac. Salud Pública [online], 30(1), 38-44.

Trull, T.J., E.J. Phares, 2003. Psicología clínica, conceptos, métodos y aspectos prácticos de la profesión. México: Thomson Learning, $580 \mathrm{pp}$.

Wartski Patiño, C.I., E. Cárdenas Pachón, M.N. Muñoz Astudillo, M. Rodríguez Patarroyo, L.M. Rivera García, A.T. Rolón, 2011. Consumo de sustancias psicoactivas en estudiantes universitarios. Pereira, Colombia: Fundación Universitaria del Área Andina, 149 pp. 